\title{
Efficacy of metformin use to induce ovulation in women with polycystic ovarian syndrome
}

\begin{abstract}
Polycystic Ovarian Syndrome (PCOS), a common disorder of the endocrine system, is diagnosed based on inclusion criteria of an ovulation or oligo-ovulation, clinical signs of hyperandrogenism, and/or presence of polycystic ovaries on ultrasound. Many women with PCOS also have hyperinsulinemia, leading to over production of ovarian androgens. Due to this differential, the use of the insulin-sensitizing agent metformin has been explored to induce ovulation in women with PCOS. The use of metformin combined with the fertility drug clomiphene citrate (clomid) has shown to be more effective than the placebo in stimulating ovulation. While metformin has not yet gained the title of a first-line pharmacological agent for ovulation induction, research shows it has many benefits in PCOS treatment.
\end{abstract}

Keywords: hyperandrogenism, anovulatory infertility, gonadotrophins, oligomenorrhea, comorbidities, transvaginal scan, gonadotrophins

\author{
Special Issue - 2018
}

\author{
Elizabeth Nista Mellott, Sharon Elizabeth \\ Metcalfe, Tonya L Hensley \\ Western Carolina University, USA
}

Correspondence: Elizabeth Nista Mellott, RN, BSN, Western Carolina University, 9 Moss Pink PI. Asheville, NC 28806, Tel (919) 924-6889, Email emmellott I@catamount.wcu.edu

Received: May 02, 2017 | Published: November 30, 2018

\section{Introduction}

Polycystic Ovarian Syndrome (PCOS) is the most common cause of anovulatory infertility in women ${ }^{1}$ making it difficult or impossible for some patients with this disorder to conceive naturally. Luckily, there are many agents available to induce ovulation in women who suffer from this syndrome. Some of these options include, but are not limited to: lifestyle modifications, selective estrogen receptor modulators, aromatase inhibitors, gonadotropins, laparoscopic ovarian drilling, assisted reproductive techniques, and insulin-sensitizing agents. ${ }^{1}$ Women with unexplained infertility (no identifiable cause of infertility on a routine evaluation) may also benefit from ovulation induction. ${ }^{2,3}$

Polycystic ovarian syndrome was first recognized by Irving Stein et $\mathrm{al}^{4}{ }^{4}$ These two gynecologists classified the disorder as heterogeneous, associated with amenorrhea, infertility, variable levels of hirsutism, and obesity in the presence of bilateral enlarged ovaries. Further inclusion criteria included the presence of "ring of pearls" on the ovary, or small cysts ranging from $2-8 \mathrm{~mm}$ in diameter. Rotterdam symptomatic criteria maintains that two out of three signs must be present for PCOS to be diagnosed; an ovulation or oligo-ovulation, clinical and/or biochemical signs of hyperandrogenism, and/or presence of polycystic ovaries on ultrasound. ${ }^{5}$ According to Bates et $\mathrm{al}, 2,3$ the most common symptom of PCOS is menstrual irregularities, with $80 \%$ of women reporting this as a symptom. Oligomenorrhea is more commonly reported than amenorrhea, and usually results from anovulatory estrogen breakthrough bleeding after a prolonged period without ovulation to produce endogenous progesterone. ${ }^{2,3}$ Metformin has been suggested in PCOS treatment to manage insulin resistance and hyperglycemia. Besides its metabolic effects, metformin has been shown to improve ovulation, pregnancy and live birth rates in PCOS patients. ${ }^{6}$

As recently as ten years ago, the use of metformin for ovulatory dysfunction was highly controversial, with clomiphene citrate (Clomid) accepted universally as the first-choice medication for ovulation induction in women with PCOS. It is only lately that it has become a widely accepted method of inducing ovulation and improving pregnancy rates. ${ }^{7}$ PCOS currently accounts for approximately $70 \%$ of all anovulatory-related infertility, and affects approximately $10 \%$ of the population. ${ }^{7}$ Though evidence suggests metformin is beneficial for some, many argue that since PCOS can manifest so differently from patient to patient, efficacy seems to differ between subgroups.

For example, some literature suggests that women who have PCOS with a BMI between 27-35 seem to have a better benefit from metformin than those who fall within a more normal weight range. ${ }^{5}$ The hope was that metformin would improve insulin sensitivity in these women, normalizing the hormonal environment, and in turn cause a return to regular ovulation and menstruation. ${ }^{8}$ In the article estimated that almost $50 \%$ of women with PCOS are obese. McCartney et al. ${ }^{9}$ discuss that lifestyle modification is important for patients who are overweight and obese, and in those with coexisting metabolic disorders. ${ }^{9}$ An additional study found that adding metformin could have a benefit on abdominal fat mass as in a decrease of ovarian stromal area irrespective from insulin resistance. ${ }^{10}$ This evidence further illustrates the benefit metformin could have towards weight loss, in turn adding fertility advantage.

Li et al. ${ }^{11}$ found that for women who are not obese, clomid should be used as the first line treatment for an ovulation, however metformin and clomid combined may be considered in a subgroup of women with PCOS who are obese or resistant to clomid. ${ }^{11}$ In their article, Propst et al. ${ }^{2}$ explore the fact that lifestyle changes to encourage weight loss are first line and initial therapy for overweight and obese patients, with clomid considered an alternative. They go on to suggest that letrozole, gonadotropins, and metformin are strong options in women who fail to ovulate on clomid alone. ${ }^{2}$

\section{Method}

An integrative review of the literature through Western Carolina University's Hunter Library online database was conducted using Cochrane Database of Systematic Reviews, Academic Search Premier, Cumulative Index of Nursing and Allied Health (CINAHL), EBSCO's Electronic Journal Service (EJS), and the National Library 
of Medicine (Pub Med). An integrative literature review is a form of research that critiques, reviews and synthesizes literature in an integrative way that allows new perspectives on the topic to be created. For the purpose of this paper, Cochrane was used to discover systematic reviews, ensuring the highest level of evidence was included. CINAHL, EJS and Pub Med were also thoroughly explored to obtain Evidence Synthesis, Randomized Controlled Trials (RCTs), and cohort studies that would satisfactorily support this topic.

When searching for data, much concern was taken to ensure only pertinent articles were included for consideration. As metformin is a drug that can be used for other comorbidities besides PCOS, it was imperative that research focused only on this one particular disease process. Keywords searched included metformin, polycystic ovarian syndrome (PCOS), an ovulation, ovulation induction, hyperandrogenism, oligomenorrhea, amenorrhea, clomiphene citrate and metformin combination therapy. From this search, over 500 articles were derived, and were subsequently included or eliminated based on inclusion criteria.

Inclusion criteria included articles that focused on PCOS with specific use of metformin therapy. Only those articles that studied subjects between the ages of 12-40 and were published within the last five years were considered. Articles were excluded if their focus was on metformin related to other disease processes (such as Type 2 Diabetes Mellitus), clomid alone for ovulation induction, or PCOS alone. As a result, forty-two articles were included for review, with five being systematic reviews.

\section{Findings}

\section{Metformin use in PCOS}

Metformin is a buguanide that acts to decrease insulin levels reduce insulin resistance, and lower free circulating insulin and androgens in the bloodstream, thus resulting in improvement of the clinical sequelae of PCOS. ${ }^{12}$ Metformin has been shown to improve ovulation rates in anovulatory women, contribute to weight reduction, and also lessen menstrual irregularities in women with PCOS. Metformin was first reported as a treatment for PCOS in 1994. ${ }^{12}$ According to the Janci et al, ${ }^{13}$ metformin has shown many beneficial effects in women with PCOS, including improvement in regulating the menstrual cycle, rate of ovulation, fertility, hirsuitism, and weight. ${ }^{13}$ The authors also go on to suggest that metformin may also decrease cardiovascular risk associated with diabetes and could also have this benefit in women with PCOS. Metformin treatment also enhances both adiponectin activity and insulin sensitivity, resulting in a less hyper androgenic state in PCOS patients. ${ }^{14}$

In the study conducted by Wahab et al, ${ }^{7}$ thirty-five female patients between the ages of 20-35, all with a diagnosis of PCOS and abnormal laboratory blood levels signifying confirmation of this disorder, were given $850 \mathrm{mg}$ of metformin twice a day for six months. Laboratory values that were part of the inclusion criteria included LH: FSH, serum prolactin, serum testosterone, serum insulin levels, and random blood sugar. All subjects also underwent a transvaginal scan. Subjects underwent clinical reassessment every three months, and compliance was ensured by providing education to the patients regarding the use of metformin at every follow-up visit. Final assessment was completed after two years, all laboratory values were re-drawn for comparison and transvaginal scan was repeated. The results of this study showed improvement in all laboratory values after two years on metformin, it also showed that metformin helped with weight reduction with an average weight loss of $4.7 \mathrm{~kg} .^{7}$ The study concluded that metformin is an effective drug to improve menstrual irregularities, LH: FSH, and even testosterone, but did not show any improvement in clinical hyperandrogenism. ${ }^{7}$ Kulshreshtha et al. ${ }^{15}$ also found that patients on metformin reported a greater reduction in body weight, as well as a significant reduction in one and two hour glucose and insulin levels.

Wagan et $\mathrm{al}^{4}{ }^{4}$ explore the effects of metformin therapy in achieving menstrual regularity, pregnancy, weight reduction, and hyperinsulinemia in patients with PCOS. This study classified their subjects into four groups based on Body Mass Index (Group 1 BMI $<25$, Group 2 BMI 25-30, Group 3 BMI 31-35, and Group 4 BMI $>35$ ). Subjects were started on a dose of $250 \mathrm{mg}$ metformin daily, and then increased gradually to $500 \mathrm{mg}$ three times a day. Patients were also included to follow a healthy diet and to exercise regularly. This study was reassessed after six months and in subjects who had failed to conceive on clomiphene citrate (Clomid) was added for three cycles. Of the sixty-three patients in the study, 10 conceived within six months and 20 conceived within one year with 13 remaining infertile. As a result of these findings, this study concluded that metformin therapy significantly increased the ovulation and conception rate. ${ }^{4}$ Another study disagreed with these findings, concluding that metformin alone has limited benefits in improving live birth rates. ${ }^{16,17}$

The study conducted by Sanoee et al..$^{18}$ also noticed a significant decrease in BMI after treatment with $1500 \mathrm{mg}$ metformin daily, as well as significant increase of FSH and decrease of LH, DHEAS, 17OHP, estradiol and testosterone. They also noted a significant increase in menstrual regularities among subjects, with up to $65 \%$ of the women reporting menstrual irregularities prior to metformin initiation reporting more regular menses. This study concluded that metformin led to reduction of ovarian volume, as well as hyperandrogenemia in women with clinical PCOS. These findings are in agreement with those of Qureshi et al. ${ }^{5}$ who also concluded from their study that metformin improves the menstrual cycle and irregularities, showing positive results in $82 \%$ of their 329 female subjects. They went on to determine that metformin is successful in treatment of PCOS in adjunct with lifestyle adjustments (such as diet and exercise) in overweight and obese patients Qureshi et al. ${ }^{5}$

Another study conducted by Morin-Papunen et al. ${ }^{19}$ found that metformin plus standard infertility treatment increased the chance of pregnancy 1.6 times. The purpose of this study was to investigate whether metformin decreased the early miscarriage rate and/or improved pregnancy and live birth rates in women with PCOS. They went on to conclude that metformin especially seems to benefit obese women with PCOS who have received three months of pre-treatment with metformin, and works in combination with other fertility drugs (unspecified) in inducing ovulation. ${ }^{19}$

Talaulikar et al. ${ }^{20}$ explored metformin use in women with PCOS and concluded that metformin also forms one of the management options in women who need anti androgen therapy without the need for contraception at the same time. ${ }^{20}$ The authors also concluded that women who suffer from PCOS and are undergoing in vitro fertilization are likely to benefit from metformin use to reduce their risk of ovarian hyper stimulation therapy. ${ }^{20}$

Rice et al. ${ }^{21}$ found that while metformin did markedly reduce FSH, it did not reduce aromatase expression and activity, concluding that there could be implications for the use of metformin in the treatment of 
anovulatory women with PCOS. Richard S Legro et al. ${ }^{16,17}$ concluded that while metformin is surrounded by a lot of "hype" as an up and coming fertility drug, head-to-head studies comparing metformin and clomid have consistently shown that pregnancy and live birth weights are significantly lower with metformin alone Legro et al, ${ }^{16,17}$ but does go on to state that meta-analysis has shown an increase in pregnancy and ovulation rates on metformin compared to that of the placebo.

The systematic review conducted by Kollmann et al. ${ }^{22}$ had different conclusions than the majority. They included 66 studies in their review of current evidence regarding the efficacy of strategies aimed at improving assisted reproductive techniques in women with PCOS, and measured the primary outcomes of live birth/ongoing pregnancy and ovarian hyper stimulation syndrome. Secondary outcomes were clinical pregnancy and miscarriage rates. They concluded that there is low-quality evidence pointing to a benefit of metformin supplementation on clinical pregnancy and live birth rates. ${ }^{22}$

Lastly, Sohrevardi et al. ${ }^{23}$ attempted to determine the effect of metformin and pioglitazone on clinical, hormonal and metabolic parameters in women with PCOS. They concluded that insulinsensitizing drugs induce beneficial effects in insulin resistance and menstrual cycle, but only metformin aided in reducing hyperandrogenemia in women with PCOS. ${ }^{23}$ These findings further evidence the fact that metformin has real potential in eliminating clinical signs and symptoms of PCOS

\section{Metformin and clomiphene citrate}

Another topic that is explored in the literature is that of using metformin combined with clomiphene citrate, better known as Clomid, for ovulation induction in women with PCOS. Knowing that metformin and clomid could each successfully induce ovulation in anovulatory women on their own prompted many to research the effects of using the two drugs simultaneously for peak results. Palomba et al. $^{24}$ found that in patients with PCOS, metformin reportedly improved ovulatory menstrual cyclicity and clinical pregnancy rate, sensitized patients resistant to clomiphene citrate, and optimized the efficacy of other pharmaceutical agents in inducing ovulation. ${ }^{24}$ Currently, clomid is considered a first-line pharmacological therapy for ovulation induction, whereas metformin has yet to gain that title for itself. Clomid remains the most used medication for inducing ovulation in anovulatory women..$^{25}$

Clomid is an anti-estrogen that competes for estrogen receptors centrally, thereby releasing the hypothalamus and pituitary from the estrogenic negative feedback and increasing the discharge of follicle stimulating hormone, leading to follicular growth and maturation. ${ }^{26}$ Interestingly enough, approximately $15-40 \%$ of patients with PCOS show resistance to clomid and continue to remain anovlatory following treatment with $150 \mathrm{mg}$ per day for three successive months. ${ }^{26}$ Recommended options for those with clomid-resistant PCOS include gonadotrophins, letrozole and laparoscopic ovarian diathermy. ${ }^{26}$

Since insulin resistance is a primary factor in the etiology of PCOS and also a large contributor to the cause of citrate-resistant PCOS, it can be used in combination with clomid (as well as on its own, as discussed above) to induce ovulation. Hashim et al. ${ }^{26}$ explore in detail the affects of combining the use of these two pharmaceutical agents. This particular study had inclusion criteria that allowed only infertile patients with clomiphene-citrate resistant PCOS to be included. They also only researched Randomized Control Trials. Twelve studies met all inclusion criteria and were included in the systematic review. ${ }^{26}$
The purpose of this review was to compare the effects of metformin and clomid combined therapy to those of other methods of ovulation induction in clomid-resistant PCOS, including gonadotrophins, laparoscopic ovarian drilling, letrozole (a third-generation aromatase inhibitor), and natural insulin sensitizers. ${ }^{26}$ Primary clinical outcomes of this study included the rate of ovulation (confirmed by serum progesterone on cycle days 21-23, reports of regular menses, or transvaginal ultrasound), and clinical pregnancy rate as defined by the presence of a gestational sac and fetal heartbeat on 7 week ultrasound scan. ${ }^{26}$ The results of the study were varied, with three of the trials showing evidence of significantly fewer ovulations following metformin and clomid compared with the use of gonadotrophins. Three trials showed no significant difference in ovulation rates between metformin and clomid and aromatase inhibitors, and one trial showed no significant difference when metformin and clomid were compared with laparoscopic ovarian drilling. Only one trial showed a significant benefit in favor of the combination of metformin and clomiphene over a natural insulin sensitizer. ${ }^{26}$

Hashim et al. ${ }^{26}$ concluded that per their evaluation of randomized control trials of women with clomid resistant PCOS, their data does not show significant differences in ovulation rates from the use of metformin and clomid combined compared to different treatment options available. Additionally, Hashim et al ${ }^{26}$ does site a specific study conducted by Siebert et al. ${ }^{27}$ in which it was found that metformin and clomid combined did significantly increase the likelihood of ovulation in comparison with clomid and placebo, suggesting that metformin does have a benefit in those with clomid-resistant PCOS. ${ }^{26}$

In the study conducted by Ayaz et al., ${ }^{28}$ forty-two subjects were divided into two groups, A and B respectively. Twenty-one females were assigned to group A where they were given clomid $50 \mathrm{mg}$ and metformin $500 \mathrm{mg}$ combined. The remaining women were placed in group B where they received clomid $50 \mathrm{mg}$ alone. In this study, the results showed that metformin and clomid when used in combination yielded impressive results. Group A achieved a higher rate of regular menstrual cycles and a higher ovulatory response. More subjects from group A conceive, and they also had a higher rate of conception. Overall, this study concluded that the combination of metformin and clomiphene citrate significantly regulated the menstrual cycle and increased ovulation and pregnancy rates in participants. They even suggest that this pharmacologic combination should be considered a first-line therapy for ovulation induction in women with PCOS. ${ }^{28}$

A randomized controlled trial conducted in India also found clomid + metformin to be more effective together than independently. ${ }^{29}$ They found rates of ovulation to be $56.2 \%$ in clomid alone, $62.5 \%$ in metformin alone, and $83.3 \%$ with the combination therapy. They concluded that metformin was as good as clomid in terms of live birth rate and that the combination gave the highest rates of ovulation and live births. ${ }^{29}$

In the study conducted by Costello et al., ${ }^{12}$ a Cochrane systematic review and meta-analysis of RCT's comparing metformin with placebo or no treatment in women with PCOS showed that metformin improved ovulation rates and clinical pregnancy rates but not live birth rates. ${ }^{12}$ They also concluded that the addition of metformin to clomiphene citrate is superior to clomiphene citrate alone in women who appear resistant. ${ }^{21}$

The study by Elgafor et al. ${ }^{30}$ explored not only metformin, but also letrozole and bilateral ovarian drilling, and concluded that they are 
similarly effective as second-line treatment for women with PCOS who were not able to conceive with clomid alone. Laparoscopic ovarian drilling is a single procedure that has minimal morbidity and can lead to consecutive ovulations with low risk of multiple pregnancies. The majority of patients seem to respond well to LOD, with only $20-30 \%$ of women failing to respond. Since the mechanism of action of LOD is not fully understood, it is not clear why some patients do not respond well to the treatment while others do. ${ }^{31}$ Letrozole is a selective aromatase inhibitor that works by decreasing estrogen production without affecting receptors, in turn reducing the negative feedback on the hypothalamus and pituitary and stimulating FSH release. ${ }^{30}$

Bjelica et al. ${ }^{32}$ also explored metformin use as well as metformin and clomid combined. They formed an algorithm of ovulation induction that illustrates the entire process of infertility and PCOS, with the first piece being that modification of lifestyle combined with use of metformin, aimed at optimizing body mass index and eliminating insulin resistance, should be the first line treatment for women with elevated body mass index. The second step of the algorithm stated that clomiphene citrate should still be used as firstline therapy for patients with PCOS. ${ }^{32}$

Bjelica et al. ${ }^{32}$ also states that the third part to the puzzle suggests that metformin and clomid combined will increase the chances of ovulation and gestation and can be considered from the beginning of treatment, and part four suggests that application of aromatase inhibitors (such as letrozole) can substitute clomid as first line treatment, though evidence is still insufficiently strong. The fifth tier of the algorithm states that application of gonadotropins and laparoscopic ovarian drilling should be second line treatment, with the sixth and final piece stating that assisted reproductive technics (such as Intrauterine Insemination and/ or In vitro Fertilization) should be considered if pregnancy does not occur after six cycles of induction with gonadotropins that is after six months following laparoscopic ovarian drilling, or when there are some associated causes of infertility. ${ }^{32}$

\section{Discussion}

The use of metformin to treat PCOS is still a relatively new topic of discussion, however current research has found that metformin is more effective than the placebo in inducing ovulation. ${ }^{16,17}$ Weight reduction and lifestyle modification should be considered first-line treatment for BMI and cardiovascular risk reduction Propst et al., 2,3 but metformin can be a suitable alternative for obese or overweight women with insulin resistance. ${ }^{5,11}$ Metformin is the only insulinsensitizing drug that has succeeded in decreasing hyperandrogenemia in women with PCOS Mojtaba Sohrevardi et $\mathrm{al}^{23}$ and has also been shown to decrease body weight $\mathrm{Du}$ et al. ${ }^{31}$ as well as to regulate the menstrual cycle. ${ }^{18}$

Metformin and clomiphene-citrate combined have also been shown to be more effective than the placebo in inducing ovulation in women with clomid-resistant PCOS. ${ }^{12}$ The combinations of the two drugs showed the highest percentage of ovulation and live pregnancy rates amongst women with combined rates as high as $83.3 \%{ }^{29}$ Metformin and clomid combined will increase the chances of ovulation and gestation and can be considered from the beginning of treatment. ${ }^{32}$

A Cochrane systematic review and meta-analysis of RCT's comparing metformin with placebo or no treatment in women with
PCOS showed that metformin improved ovulation rates and clinical pregnancy rates but not live birth rates. They further concluded that the addition of metformin to clomiphene citrate is superior to clomiphene citrate alone in women who appear resistant. ${ }^{12}$ Metformin and clomid combined did significantly increase the likelihood of ovulation in comparison with clomid and the placebo in the study conducted by ${ }^{26}$

\section{Conclusion}

In summation, insulin sensitizers, such as metformin, increase insulin sensitivity and improve ovulation. ${ }^{33,34}$ Patients on metformin reported a greater reduction in body weight, as well as a significant reduction in one and two hour glucose and insulin levels. ${ }^{15}$ Improvements in BMI have also been observed, as well as a significant increase in menstrual regularities among subjects. ${ }^{18}$ Metformin is an appropriate alternative to decrease menstrual regularities and improve weight reduction in women with PCOS, but evidence does not suggest it should be considered a first-line pharmaceutical agent for ovulation induction at this time. ${ }^{32}$

\section{Acknowledgements}

None.

\section{Conflict of interest}

The author declares no conflict of interest.

\section{References}

1. Perales-Puchalt A, Legro R S. Ovulation induction in women with polycystic ovary syndrome. Steroids. 2013;78(8):767-772.

2. Propst A M, Bates G W Jr. Evaluation and treatment of anovulatory andunexplained infertility. Obstet Gynecol Clin North Am. 2012;39(4):507-519.

3. Bates GW Jr, Propst AM. Polycystic Ovarian Syndrome Management Options. Obstet Gynecol Clin North Am. 2012;39(4):494-506.

4. Wagan F, Suhail M A, Laghari A. Polycystic ovarian syndrome: Response to metformin therapy. Pakistan Journal of Medical Science. 2011;27(5):1038-1041.

5. Qureshi R T, Rahim F Haidar G. Polycystic Ovarian Disease; Impact of Metformin On Fertility In Women. Professional Medical Journal. 2016;23(7):775-779.

6. DiPietro M, Parborell F, Irusta G, et al. Metformin regulates ovarian angiogenesis and follicular development in a female polycystic ovary syndrome rat model. Endocrinology. 2015;156(4):1453-1463.

7. Wahab S, Zahoor F, Karim R. Role of Metformin In Polycysticovarian Syndrome. Journal of Postgraduate Medical Institute. 2013:27(2).

8. Messinis I E, Messini C I, Anifandis G, et al. Polycystic ovariesand obesity. Best Pract Res Clin Obstet Gynaecol. 2015;29(4):479-488.

9. McCartney C R MD, Marshall J C MD. Polycystic Ovary Syndrome. The New England Journal of Medicine. 2016;375:54-64.

10. Cakiroglu Y, Vural B, Isgoren S. The effects of drospirenoneethinylestradiol and drospirenone-ethinyl estradiol metformin on ovarian ultrasonographic markers, body fat mass index, leptin, and ghrelin. Arch Gynecol Obstet. 2013;288(1):213-220.

11. Li R H, Ng E H. Management of anovulatory infertility. Best Practice \& Research Clinical Obstetrics and Gynaecology. 2012;26(6):757-768. 
12. Costello M F, Misso M L, Wong J, et al. The treatment of infertility in polycystic ovary syndrome: a brief update. Aust N Z J Obstet Gynaecol. 2012;52(4):400-403.

13. Jancy M M, ARNP, CDE, et al. Polycystic Ovarian Syndrome: Metformin or Thiazolidinediones for Cardiovascular Risk Reduction? Diabetes Spectrum. 2012;25(4):229-237.

14. Hamed H. Role of adiponectin and its receptor in prediction of reproductiveoutcome of metformin treatment in patients with polycystic ovarian syndrome. Journal of Obstetrics and Gynecology. 2013;39(12):1596-1603.

15. Kulshreshtha B, Gupta N, Ganie M A, et al. Effect of metforminand spironolactone therapy on OGTT in patients with polycystic ovarian syndrome - a retrospective analysis. Gynecol Endocrinol. 2012;28(10):823-826.

16. Balen A H, Morley L C, Misso M, et al. The management of anovulatory infertility in women with polycystic ovary syndrome: an analysis of the evidence to support the development of global WHO guidance. Hum Reprod Update. 2016;22(6):687-708.

17. Legro R S. Ovulation induction in polycystic ovary syndrome: Current options. Best Practice and Research Clinical Obstetrics and Gynecology. 2016;37:152-159.

18. FarimaniSanoee M, Negha N, Rabiee $\mathrm{S}$, et al. Metformin therapydecreases hyperandrogenism and ovarian volume in women with polycystic ovary syndrome. Iran J Med Sci. 2011;36(2):90-95.

19. Morin-Papunen L, Rantala A S, Unkila-Kallio L, et al. Metformin improves pregnancy and live-birth rates in women with polycystic ovary syndrome (PCOS): a multicenter, double-blind, placebocontrolled randomized trial. The Journal of Clinical Endocrinology and Metabolism. 2012;97(5):1492-1500.

20. Sinai Talaulikar, Vikram MD, MRCOG, et al. Role of Metformin in Womens Health: Review of Its Current Place in Clinical Practice and Emerging Indications for Future. Obstetrical and Gynecological Survey. 2016;71(5):307-317.

21. Rice S, Elia A, Jawad Z, et al. Metformin Inhibits FollicleStimulating Hormone (FSH) Action in Human Granulosa Cells: Relevance to Polycystic Ovary Syndrome. J Clin Endocrinol Metab. 2013;98(9):E1491-E1500.

22. Kollmann M, Martins WP, Lima ML, et al. Strategies for improving outcome of assisted reproduction in women with polycystic ovary syndrome: systematic review and meta-analysis. Ultrasound Obstet Gynecol. 2016;48(6):709-718.

23. Sohrevardi SM, Nosouhi F, Khalilzade SH, et al. Evaluating the effect of insulin sensitizers metformin and pioglitazone alone and in combination on women with polycystic ovary syndrome: An RCT. Int J Reprod Med. 2016;14(12):743-754.
24. Palomba S, Falbo A, La Sala GB. Effects of metformin in women withpolycystic ovary syndrome treated with gonadotrophins for in vitro fertilisation and intracytoplasmic sperm injection cycles: a systematic review and meta-analysis of randomised controlled trials. BJOG. 2013;120(3):267-276.

25. Bhagavath B, Carson SA. Ovulation induction in women with polycysticovary syndrome: an update. Am J Obstet Gynecol. 2012;206(3):195-198.

26. Abu Hashim H, Foda O, Ghayaty E. Combined metformin-clomiphene in clomiphene-resistant polycystic ovary syndrome: a systematic review and meta-analysis of randomized controlled trials. Acta Obstet Gynecol Scand. 2015;94(9):921-930.

27. Siebert TI, Kruger TF, Steyn DW, et al. Is the addition of metforminefficacious in the treatment of clomiphene citrate-resistant patients with polycystic ovary syndrome? A structured literature review. Fertil Steril. 2006;86(5):1432-1437.

28. Ayaz A, Alwan Y, Farooq MU. Efficacy of combined metforminclomiphene citrate in comparison with clomiphene citrate alone in infertile women with polycystic ovarian syndrome (PCOS). $J$ Med Life. 2013;6(2):199-201.

29. Karim S, Sanchita S. Clomiphene citrate, metformin or a combination ofboth as the first line ovulation induction drug for Asian Indian women with polycystic ovarian syndrome: A randomized controlled trial. J Hum Reprod Sci. 2015;8(4):197-201.

30. Elgafor A. Efficacy of combined metformin-letrozole in comparison withbilateral ovarian drilling in clomiphene-resistant infertile women with polycystic ovarian syndrome. Arch Gynecol Obstet. 2013;288(1):119-123.

31. Dudhat A. Laparoscopic Ovarian Drilling and its Outcome. International Journal of Collaborative Research on Internal Medicine \& Public Health. 2012;4(12):2075-2083.

32. Bjelica A, Bjelanovic J, Millc N, et al. Algorithm of ovulation induction in patients with polycistic ovary syndrome. Med Pregl. 2016;69(12):25-30.

33. Saha L, Kaur S, Saha PK. Pharmacotherapy of polycystic ovarysyndrome - an update. Fundam Clin Pharmacol. 2012;26(1):54 62.

34. Al-Mukhtar Entisar J, Al-Zubaid, Adeeb A, et al. Finasteride (plusOral Contraceptive pill) vs Metformin in Treatment of Polycystic Ovary Syndrome-Related Infertility: a Prospective Randomized Trial. Iraqi Journal of Medical Sciences. 2013;11(1):50-58. 